\title{
SUR L'IMPORTANCE DES DIPTẼRES DANS L'ENVIRONNEMENT DE QUELQUES COURS D'EAU DES PYRÉNÉES
}

\author{
par A. Thомаs \\ 1. - INTRODUCTION ${ }^{1}$.
}

Les Diptères constituent l'un des groupes les plus mal connus parmi les invertébrés totalement ou partiellement aquatiques. La difficulté de leur étude tient surtout à l'extrême diversité de leurs formes et de leurs modes de vie :

- on compte, en Europe Occidentale, plus de 80 familles totalisant probablement une vingtaine de milliers d'espèces;

- en dehors des espèces parasites, les larves de Diptères vivent dans des milieux aussi variés que les cours d'eau de haute montagne, la terre humide, les substances organiques en décomposition ou les mares de pétrole.

En outre, la plupart des familles de Diptères ne sont pas exclusivement aquatiques. Il est fréquent de trouver chez une même famille, un nombre variable d'espèces «subaquatiques» et «terrestres» aux côtés d'espèces «aquatiques». Ces dernières peuvent d'ailleurs souvent supporter un assèchement plus ou moins long de leur habitat.

En ce qui concerne l'étude de la faune des eaux courantes, Jeux questions principales se posent :

1) Quelles sont les familles de Diptères possédant des espèces aquatiques au moins pendant une partie de leur cycle de développement? Plusieurs études de synthèse ont été publiées à ce sujet [Bertrand 1954, VaILlant 1955, Usinger et coll. 1963, Illies et coll. 1967], mais de nombreuses précisions sont encore à apporter sur ce point [ILLIES 1967].

2) Dans l'économie d'un cours d'eau, quelle est l'importance quantitative des espèces aquatiques de chaque famille par rapport aux familles mieux connues comme les Chironomidae ou les Simuliidae? Nous ne possédons que très peu de données d'ensemble sur ce point. E. ANGELIEr [1959] cite près de 200 espèces de Nématocères et de Brachycères capturées près de divers cours d'eau corses (principalement d'après des déterminations de F. W. Edwards et de F. Vaillant).

1. Je remercie MM. F. Vaillant de Grenoble et B. Mannheims de Bonn pour l'aide qu'ils m'ont apportée. 
L'objet de cette note est de préciser qualitativement et quantitativement, l'importance relative des Diptères adultes dans l'environnement des cours d'eau. Mes captures proviennent d'une quarantaine de ruisseaux et torrents du Bassin de la Vallée d'Aure (Hautes-Pyrénées) et de cinq cours d'eau des Prépyrénées et des environs de Toulouse. Ces cours d'eau, échelonnés entre 150 et 2400 m d'altitude, appartiennent au « rhithron».

\section{2. - CONDITIONS DE CAPTURE.}

- instrument utilisé pour les Diptères : le filet à mailles fines;

- l'opérateur a été le même pendant toutes les chasses;

- plus de cent chasses ont été effectuées pendant toute l'année entre dix heures du matin et la tombée de la nuit;

- aucune imago n'a été capturée à plus de cinq mètres de l'eau.

\begin{tabular}{|c|c|c|c|}
\hline & Famille & $\begin{array}{l}\text { NOMBRE } \\
\text { D'IMAGOS } \\
\text { CAPTURÉES }\end{array}$ & $\%$ DU TOTAI \\
\hline $\begin{array}{l}\text { Classe } 1 \text { : } \\
\text { nombreux ou très } \\
\text { nombreux } \\
(2500 \text { à } 400)\end{array}$ & $\begin{array}{l}\text { 1. Chironomidae } \\
\text { 2. Iimoniidae } \\
\text { 3. Cypselidae } \\
\text { 4. Psychodidae } \\
\text { 5. Lonchopteridae } \\
\text { 6. Ceratopogonidae } \\
\text { 7. Sciaridae }\end{array}$ & $\begin{array}{r}2467 \\
843 \\
635 \\
634 \\
574 \\
441 \\
424\end{array}$ & $\begin{array}{r}30,8 \\
10,5 \\
7,9 \\
7,9 \\
7,2 \\
5,5 \\
5,3\end{array}$ \\
\hline $\begin{array}{l}\text { Classe } 2 \text { : } \\
\text { assez nom- } \\
\text { breux } \\
(400 \text { a } 100)\end{array}$ & $\begin{array}{l}\text { 8. Ephydridae } \\
\text { 9. Tipulidae } \\
\text { 10. Dolichopodidae } \\
\text { 11. Phoridae } \\
\text { 12. Trichoceridae } \\
\text { 13. Simuliidae } \\
\text { 14. Cecidomyiidae } \\
\text { 15. Dixidae } \\
\text { 16. Blepharoceridac } \\
\text { 17. Empididae } \\
\text { Hemerodromiinae }\end{array}$ & $\begin{array}{l}292 \\
258 \\
238 \\
208 \\
148 \\
130 \\
130 \\
129 \\
109 \\
\\
101\end{array}$ & $\begin{array}{l}3,6 \\
3,2 \\
3 \\
2,6 \\
1,8 \\
1,6 \\
1,6 \\
1,6 \\
1,4 \\
1,3\end{array}$ \\
\hline $\begin{array}{l}\text { Classe } 3: \\
\text { peu nombreux } \\
\text { ou rares } \\
(<100)\end{array}$ & $\begin{array}{l}\text { 18. Culicidae } \\
\text { 19. Rhagionidae } \\
\text { 20. Tabanidae } \\
\text { 21. Thaumaleidae } \\
\text { 22. Syrphidae } \\
\text { Eristalinae } \\
\text { 23. Stratiomyiidae } \\
\text { 24. Tetanoceridae } \\
\text { 25. Ptychopteridae }\end{array}$ & $\begin{array}{r}76 \\
62 \\
45 \\
20 \\
\\
19 \\
18 \\
13 \\
7\end{array}$ & $\begin{array}{l}0,9 \\
0,8 \\
0,6 \\
0,2 \\
\\
0,2 \\
0,2 \\
0,15 \\
<0,1\end{array}$ \\
\hline \multicolumn{2}{|c|}{$\begin{array}{l}\text { Total général : } \\
\text { Nématocères : } \\
\text { Brachycères : }\end{array}$} & $\begin{array}{l}8014 \\
5811 \\
2203\end{array}$ & $\begin{array}{l}\text { imagos } \\
(72,5 \%) \\
(27,5 \%)\end{array}$ \\
\hline
\end{tabular}




\section{3. - FAMilles ÉTUdiées.}

25 familles possédant des espèces aquatiques ou susceptibles d'en posséder figurent dans le tableau ci-contre.

En réalité, près de 14000 individus ont été capturés, dont 9228 considérés comme aquatiques certains ou probables. Compte tenu des essaims, ce chiffre a été ramené à 8014 imagos groupées en trois classes. Les proportions relatives des différentes familles de Diptères sont concordantes d'une chasse à l'autre; ces proportions ont, pour cette raison, été traduites en pourcentages du total.

\section{Remarques.}

- La plupart des Ceratopogonidae et des Cecidomyiidae sont de si petite taille qu'ils peuvent s'échapper par des orifices d'un diamètre inférieur à $1 \mathrm{~mm}$ et le nombre indiqué peut être inférieur à Ia réalité.

- Les « essaims» capturés n'ont pas été comptés lors des pourcentages pour éviter de fausser les chiffres moyens. En pratique, les essaims - sauf chez les Empididae terrestres - sont relativement peu fréquents : il n'en a été dénombré que trois (un chez les Chironomidae, un chez les Dixidae et un chez les Lonchopteridae).

\section{Autres Diptères capturés.}

Le reste (près de 4600 imagos) est composé en grande partie de formes très hygrophiles dont les larves doivent souvent vivre en milieu humide. On remarquera en effet le nombre réduit des imagos d'espèces plus franchement «terrestres» (Bombyliidae, Asilidae, Syrphidae non Eristalinae) pourtant assez communes dans divers milieux terrestres.

Toutes les autres familles de Nématocères sont représentées au bord des eaux courantes : dans ces captures par environ 500 Mycetophilidae très hygrophiles, 120 Bibionidae, quelques rares Anisopodidae et Scatopsidae.

Les Brachycères sont cependant les plus nombreux. Parmi ceux-ci, 2250 Empididae empidiniens (en très grande majorité du genre Hilara), et 660 Muscidae. 750 individus sont des Ephydridoïdes et des Tétanocéroïdes essentiellement représentés par des Lauxaniidae, des Drosophilidae et des Chloropidae. On dénombre encore une centaine de Syrphidae non Eristalinae, autant de Sepsidae et une soixantaine de Scatophagidae. Il faut enfin citer de rares Bombyliidae, Asilidae, Therevidae, Tachinidae et Calliphoridae.

1. Les Rhamphomyia comptent quelques rares espèces aquatiques. 


\section{4. - NOTES SUR LES FAMILLES.}

\section{1. - Chironomidae.}

Proportions relatives des différentes sous-familles :

Orthocladiinae $\ldots \ldots \ldots \ldots \ldots \ldots \ldots$
$\begin{aligned} & (+ \text { quelques } \text { rares Tanypodinae) } \\ & \text { Chironominae } \ldots \ldots \ldots \ldots \ldots \ldots \ldots \ldots\end{aligned}$ 22 $\%$
Diamesinae $\ldots \ldots \ldots \ldots \ldots \ldots \ldots$

\section{2. - Limoniidae.}

Les six sous-familles sont ainsi représentées :

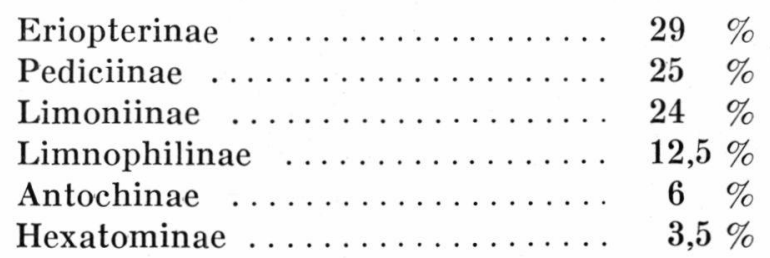

La biologie de beaucoup d'espèces est encore mal connue et le nombre de 73 cité avec prudence par P. Nielsen dans la Limnofauna Europaea [ILLIEs et coll. 1967] est certainement assez inférieur à la réalité.

La systématique des Pediciinae, Limnophilinae et Hexatominae demande une révision à l'échelle européenne.

\section{3. - Cypselidae ( $=$ Borboridae $=$ Sphaeroceridae $)$.}

Si l'on utilise la classification simplifiée de SÉGUY [1934] en trois genres au sens large, les pourcentages relatifs obtenus sont les suivants :

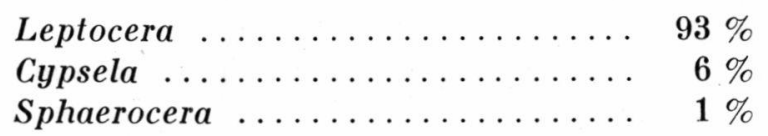

Beaucoup de larves de Cypselidae vivent sur les matières organiques en décomposition. Néanmoins, aucune chasse n'a été effectuée sans capture de Leptocera. Ce sont, semble-t-il, toujours les mêmes espèces (en nombre réduit) qu'on rencontre au bord des eaux courantes. M. F. VAILlant (comm. verb.) a d'autre part obtenu des Cypselidae par élevage à partir de mousses aquatiques. Ce fait ne semble pas avoir été signalé (le groupe est absent de la Limnofauna Europaea). 


\section{4. - Psychodidae.}

Groupe comptant dans ces captures près de $8 \%$ du total. Les larves sont très souvent aquatiques et se rencontrent en grand nombre dans différents milieux. Dans les ruisseaux fortement peuplés de la Vallée d'Aure vers $1200 \mathrm{~m}$, leur nombre est comparable à celui des Simuliidae. Dans les mousses aquatiques des mêmes ruisseaux, leur nombre peut dépasser celui des Chironomidae.

\section{5. - Lonchopteridae.}

La biologie des larves de cette famille est mal connue de même que ses affinités phylétiques. La présence d'imagos est constante dans les stations prospectées. Les larves sont probablement toutes terrestres.

\section{6. -- Ceratopogonidae.}

Sauf pour le genre Culicoides, très étudié par les laboratoires d'entomologie médicale, la systématique du groupe doit être révisée.

Genres capturés :

\begin{tabular}{|c|c|}
\hline Atrichopogon & $38 \%$ \\
\hline Culicoides & $38 \%$ \\
\hline Forcipomyia & $14 \%$ \\
\hline Palpomyia . & $6 \%$ \\
\hline Dasyhelea & 3 \\
\hline Helea + Stilobezzia & 1 \\
\hline
\end{tabular}

\section{7. - Sciaridae.}

A l'exception de plusieurs espèces pétrimadicoles citées par VaILLANT [1955], aucun travail de Limnologie ne semble indiquer la présence de Sciaridae en milieu aquatique. Des élevages ont cependant montré que les torrents pyrénéens peuvent renfermer quelques espèces de Sciaridae.

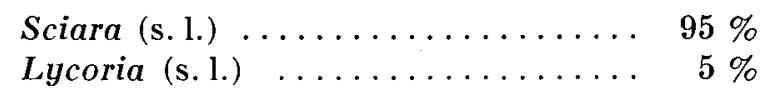

Tous les individus obtenus par élevage étaient des Sciara s. l.

\section{8. - Ephydridae.}

Petits Brachycères dont la biologie est surtout connue dans les milieux saumâtres ou salés [DAHL 1959].

L'espèce Hydrellia griseola Fallén est de loin la plus répandue : on la rencontre à toutes les stations prospectées. On sait que sa larve mine de nombreux végétaux aquatiques ou non, dont des Graminées [SÉguy 1934]. 


\section{Hydrellia griseola Fallén ........ $61 \%$ \\ Napaea .................... $24 \%$ \\ $\therefore$ Scatella stagnalis Fallén .......... $3 \%$ \\ Notiphila+Hydrina+Discocerina, etc. $12 \%$}

\section{9. - Tipulidae.}

Numériquement, les Tipulidae n'occupent que la $9^{e}$ place dans ce classement. Au point de vue biomasse, la grande taille des larves en fait cependant l'un des groupes de Diptères les plus importants dans les eaux courantes.

40 espèces ont été identifiées dans ce matériel par M. B. MaNNHEIMs; deux des plus importantes numériquement sont :

Tipula (Yamatotipula) lateralis Meigen .. $10 \%$

Tipula (Emodotipula) saginata Bergroth. $6 \%$

Ces deux espèces ont d'autre part été obtenues par élevage de larves prélevées à plus de $2000 \mathrm{~m}$.

\subsection{0. - Dolichopodidae.}

7 sous-familles ont été capturées.

Dolichopodinae $48 \%$ :

Hercostomus .......................... $34 \%$

Dolichopus ...................... $8 \%$

Hypophyllus (H.obscurellus Fallén) .......... $4 \%$

Ludovicius (L. dufouri Macquart, L. spectabilis Parent)

$2 \%$

Campsicneminae $32,5 \%$ :

Campsicnemus (C. curvipes Fallén, C. umbripennis Loew)

Sympycnus ( $S$. annulipes Meigen, S. cirrhipes Wa!ker)

Divers (Teuchophorus sp, Anepsiomyia flaviventris Meigen, Chrysotimus molliculus Fallén) ...... Rhaphinae $11 \%$ :

Syntormon ( $S$. pallipes Fabricius presque uniquement)

Divers (Porphyrops vandeli Thomas, Xiphandrium $\mathrm{sp}$, Eutarsus aulicus Meigen) $\ldots \ldots \ldots \ldots \ldots, 6 \ldots$ Hydrophorinae $4 \%$ :

(à peu près exclusivement Hydrophorus balticus Meigen et Liancalus virens Scopoli)

Diaphorinae $3,5 \%$ :

Argyra

$2 \%$

Divers (Chrysotus laesus Wiedemann, Melanostolus melancholicus Loew) 
Medeterinae (Medetera sp) ................ $1 \%$

Chrysosomatinae (Sciopus sp) .............. $1 \%$

\subsection{1. - Phoridae.}

On trouve à toutes les stations de chasse quelques imagos appartenant apparemment à un petit nombre d'espèces. Des formes plus ou moins franchement aquatiques ont été signalées d'Amérique du Nord et des Indes hollandaises [Usinger et coll. 1963].

Des specimens appartenant à deux sous-familles ont été capturés :

Metopininae
Phorinae $\ldots \ldots \ldots \ldots \ldots \ldots \ldots \ldots \ldots \ldots$$\quad \begin{array}{r}92 \% \\ \%\end{array}$

De récents élevages viennent de montrer que des espèces de Phoridae peuvent apparaître à haute altitude dans les torrents pyrénéens, au moins vers la fin de leur cycle de développement.

\subsection{2. - Trichoceridae.}

Cette famille n'était pas considérée comme aquatique jusqu'à la récente découverte par Valllant (comm. verb.) d'une larve dans l'Isère. Les adultes des 6 espèces capturées sont manifestement très hygrophiles.

\subsection{3. - Simuliidae.}

Un des groupes de Diptères aquatiques les plus importants. Le pourcentage indiqué n'a guère de signification car les + adultes hématophages - il y a $95 \%$ de $\%$ dans mes captures - peuvent s'éloigner des cours d'eau où vivent les larves.

\subsection{4. - Cecidomyiidae.}

VaIllant [1955] a signalé des Cecidomyiidae aquatiques (habitat pétrimadicole). Les adultes capturés ici sont cependant sans doute en grande partie parasites de Phanérogames.

\subsection{5. - Dixidae.}

Les imagos vivent souvent en groupes, parfois d'un petit nombre d'individus. Gîtes : on trouve fréquemment les imagos dans les endroits humides (sous les ponts ou au-dessus des.mousses).

\subsection{6. - Blepharoceridae.}

Une importante étude de ce groupe a récemment été effectuée en Corse par Giudicelli [1968].

Les imagos des deux sexes se capturent surtout en vol au-dessus de l'eau. 


\subsection{7. - Empididae Hemtrodromiinae.}

La méthode de capture au filet est assez peu efficace car les imagos du genre Wiedemannia sautillent constamment sur les rochers émergés en plein courant, dans la zone de balancement de l'eau, au milieu des embruns. Le pourcentage indiqué ici est certainement inférieur à la réalité.

\subsection{8, 4.22, 4.25. -- Culicidae, Syrphidae Eristalinae et Ptychopteridae.}

Les specimens de ces trois groupes, bien qu'aquatiques, ne proviennent probablement pas des cours d'eau eux-mêmes mais plutôt de petites collections d'eau voisines.

\subsection{9. - Rhagionidae.}

6 espèces ont été capturées :

Atherix marginata Fabricius ..... $39 \%$ Chrysopilus cf auratus Fabricius .... $18 \%$ Atrichops crassipes Meigen ....... $13 \%$

Rhagio lineola Fabricius ........ $13 \%$ Rhagio pandellei Gobert ........ $13 \%$ Rhagio tringarius Linné ......... $5 \%$

La Limnofauna Europaea ne mentionne qu'Atherix ibis Fabricius. Des imagos d'Atherix marginata ont été obtenues par élevages de larves prélevées en courant rapide dans un torrent de la Vallée d'Aure à $1200 \mathrm{~m}$ et dans la Garonne en amont de Toulouse. Cette dernière espèce et Chrysopilus auratus ont également été signalées par Vaillant [1955] dans l'habitat pétrimadicole. Dans les ruisseaux et torrents d'altitude inférieure à $1300 \mathrm{~m}$, fortement peuplés et peu pollués, le nombre des larves de ces Diptères peut devenir assez important ainsi probablement que leur rôle en tant que prédateurs.

\subsection{0. - Tabanidae.}

Le genre Haematopota (synonyme de Chrysozona) est cité dans l'Afon Hirnant [Hynes 1961]. On observe un fort pourcentage de ce genre au bord des cours d'eau pyrénéens :

$$
\begin{array}{ll}
\text { Chrysozona pluvialis Linné ........ } & 60 \% \\
\text { Ochrops + Atylotus + Sziladinus, etc } & 40 \%
\end{array}
$$

Presque tous les spécimens récoltés sont des $\&$ et proviennent de l'environnement de ruisseaux fréquemment traversés par des troupeaux de bovins (ce fait se remarque presque aussi nettement avec les Simuliidae et les Ceratopogonidae à la fois dans les chasses et dans les prélèvements). 


\subsection{1. - Thaumaleidae.}

VaIllant [1955] cite plusieurs espèces pétrimadicoles. Cette famille peut occuper une place importante dans les ruisseaux de faible débit, à forte pente, où une grande partie du lit est formée de pierres mouillées par capillarité. On trouve fréquemment dans ce milieu des Ephémères du genre Baetis.

\subsection{3 et 4.24 . - Stratiomyiidae et Tetanoceridae.}

Ces deux familles comptent, d'après la Limnofauna Europaea, respectivement 105 et 73 espèces aquatiques. Le nombre d"imagos capturées est cependant réduit, de même probablement que le rôle des larves dans les chaînes alimentaires de la plupart des milieux d'eau courante.

Remarque :

Deux familles sont encore citées dans la Limnofauna Europaea : les Scatophagidae et les Muscidae.

664 Muscidae ont été capturés (12 Stomoxidinae et Muscinae). Le genre Lispe Latreille ${ }^{1}$, n'a pu être mis en évidence et la famille n’a pas été incluse dans le tableau proposé.

Les 66 Scatophagidae récoltés appartiennent presque tous à la sous-famille des Scatophaginac, essentiellement connue jusqu’ici comme coprophile.

\section{5. - CONCLUSION.}

Le bilan numórique global concernant les trois principaux groupes d'imagos capturées se traduit comme suit :

$$
\begin{aligned}
& \text { Plécoptères }+ \text { Trichoptères } \ldots \ldots \ldots \ldots \quad 17 \% \\
& \text { Totalité des Diptères } \ldots \ldots \ldots \ldots \ldots \quad 83 \%
\end{aligned}
$$

Ce résultat devra être interprété avec prudence et il appelle quatre remarques :

- il ne tient pas compte du nombre des Ephémères dont les captures ne sont pas comparables;

- il ne tient pas compte non plus de la durée de vie imaginalc dans les différents groupes;

- suivant les ordres, deux méthodes de chasse ont été employées (filet et pince);

- tous les Diptères capturés ne sont évidemment pas aquatiques.

La proportion de $80 \%$ illustre bien cependant l'énorme prolifération des Diptères au bord des cours d'eau.

1. Ce genre compte [IL...1Fs et coll. 1967] 22 des 29 espèces citées. 


\section{RÉSUMÉ}

Des chasses au filet ont été effectuées afin de déterminer l'importance des Diptères adultes dans l'environnement de quelques cours d'eau pyrénéens.

1. - 25 familles de Diptères (plus de 8000 individus) ont été étudiées.

2. - Les imagos appartenant aux familles les mieux représentées Chironomidae, Limoniidae, Cypselidae, Psychodidae, Lonchopteridae, Ceratopogonidae et Sciaridae - forment environ $75 \%$ des Diptères capturés.

3. - Par rapport aux Plécoptères et Trichoptères récoltés au cours de ces chasses, les Diptères représentent environ $80 \%$ des adultes capturés.

\section{ON THE IMPORTANCE OF ADULTE DIPTERA IN THE ENVIRONMENT OF SOME PYRENEAN STREAMS}

Nettings took place in order to determine the extent of existence of adult Diptera in some pyrenean streams.

1. - 25 families of Diptera (more than 8000 specimens) were studied.

2. - The imagos belonging to the families present in the greatest number - Chironomidae, Limoniidae, Cypselidae, Psychodidae, Lonchopteridae, Ceratopogonidae and Sciaridae - constitute approximatively $75 \%$ of the Diptera captured.

3. - With respect to the Plecoptera and Tricoptera gathered during nettings the Diptera represent almost $80 \%$ of the adults captured.

\section{ÜBER DIE BEDEUTUNG DER DIPTEREN-IMAGOS IN DER UMGEBUNG EINIGER WASSERLÄUFE DER PYRENÄEN}

Um die Bedeutung der Dipteren-Imagos in der Umgebung einiger Wasserläufe der Pyrenäen zu bestimmen, wurde einige Netzfänge unternommen.

1. - 25 Dipterenfamilie (mehr als 8000 Individuen) sind untersucht worden.

2. - Die Imagines der am besten vertretenen Familie sind - Chironomidae, Limoniidae, Cypselidae, Psychodidae, Lonchopteridae, Ceratopogonidae und Sciaridae - diese bilden ca. $75 \%$ der eingefangenen Dipteren.

3. - Bezogen auf die bei diesen Fängen gesammelten Plecopteren und Trichopteren stellen die Dipteren nahezu $80 \%$ der gefangenen ausgewachsenen Tiere. 


\section{TRAVAUX CITÉS}

Angelier (E.). 1959. - Les eaux douces de la Corse et leur peuplement. Vie Milieu, Paris, suppl. $8: 1-56$.

Bertrand (H.). 1954. - Les insectes aquatiques d'Europe. Vol 2 : Trichoptères, Lépidoptères, Diptères, Hyménoptères. Encycl. ent., Lechevalier, 547 p.

DaHL (R.). 1959. - Studies on Scandinavian Ephydridae. Opusc. ent., suppl. 15 : 1-225.

Giudicelli (J.). 1968. - Recherches sur le peuplement, l'écologie et la biogéographie d'un réseau hydrographique de la Corse centrale. Thèse, C.N.R.S. : A.O. 2478,437 p.

Hynes (H. B. N.). 1961. - The invertebrate fauna of a Welsh mountain stream. Arch. Hydrobiol., 5y (3) : 344-388.

Illies (J.) et coll. 1967. - Limnofauna Europaea. Stuttgart. XV + $474 \mathrm{p}$.

SÉGuY (E.). 1934. - Diptères (Brachycères) (Muscidae acalypterae et Scatophagidae). In Faune de France. Paris. 832 p + XXVII pl.

Usinger (R. L.) et coll. 1963. - Aquatic Insects of California. Berkeley and Los Angeles. 508 p.

Vaillant (F.). 1955. - Recherches sur la faune madicole de France, de Corse et d'Afrique du Nord. Lons-le-Saunier. 257 p.

(Laboratoire d'Hydrobiologie de la Faculté des Sciences de Toulouse. Equipe de recherche associée au C.N.R.S.) 